\title{
Einleitung: Was ist Diskriminierung und was genau ist daran moralisch falsch?
}

\section{Introduction: What is Discrimination and What Makes it Morally Wrong?}

Andrea Klonschinski, Kiel

Zusammenfassung: Während wir in vielen Fällen ein intuitives Verständnis dafür haben, was gemeint ist, wenn von „Diskriminierung“ die Rede ist, zeigt sich bei näherem Hinsehen, dass eine präzise Definition des Begriffs gar nicht so einfach ist. Dasselbe gilt für die Antwort auf die Frage, was genau das spezifische moralische Übel diskriminierender Handlungen ausmacht. Nicht nur aufgrund ungeklärter Fragen im theoretischen Diskurs, sondern auch angesichts seiner gesellschaftlichen Relevanz ist eine philosophische Auseinandersetzung mit dem Thema Diskriminierung geboten. Dieser Beitrag gibt im ersten Teil einen Überblick über zentrale Themen und Kontroversen der aktuellen philosophischen Diskriminierungsdebatte. Im zweiten Teil werden die Beiträge dieses Schwerpunkts vorgestellt und in der zuvor skizzierten Debatte verortet.

Schlagwörter: Diskriminierung, Gerechtigkeit, Gruppenkriterium, implizite Vorurteile, Antidiskriminierung/Affirmative Action

Abstract: Although in most cases, we do have an intuitive understanding of what "discrimination" means, a closer look reveals that it is far from clear how this concept should be defined. The same is true when it comes to the normative-ethical evaluation of discrimination and the question of what its specific wrong making features consists in. Not only due to such open questions within the theoretical discourse, but also regarding the social relevance of discrimination, philosophical analysis of the topic seems required. In its first part, this paper offers an overview of some central issues discussed in the current philosophical debate on discrimination. The second part introduces the contributions to this special issue and relates them to the topics discussed in part one.

Keywords: Discrimination, justice, group criterion, implicit biases, affirmative action einer Creative Commons Namensnennung 4.o International Lizenz.

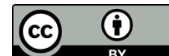




\section{Einleitung}

Seit Ausbruch der Corona-Pandemie wird von sich häufenden Diskriminierungen von asiatisch aussehenden Personen berichtet. ${ }^{1}$ So sei einer chinesischen Studentin etwa mit der Begründung, man wolle keinen Corona-Virus, eine besichtigte Wohnung nicht vermietet und einem Patienten chinesischer Herkunft die Behandlung in einer Arztpraxis verweigert worden. Mittlerweile gerät die potentielle Diskriminierung älterer oder chronisch kranker Menschen bei der Verteilung von lebensrettenden Beatmungsmöglichkeiten (vgl. Lübbe 2020) sowie eine befürchtete Verstärkung des Generationenkonflikts durch Altersdiskriminierung in den Fokus der Debatte. ${ }^{2}$ Die Berechnung des Kurzarbeitergelds, so kritisiert der Deutsche Juristinnenbund, diskriminiere zudem Frauen. ${ }^{3}$

Diese Berichte sind zunächst intuitiv ohne Weiteres verständlich; es werden hier offenbar jeweils Personen bzw. Personengruppen auf der Basis eines bestimmten Gruppenmerkmals benachteiligt. Derartige Benachteiligungen gelten allgemein als moralisch falsch und sind durch nationale und internationale Statuten verboten; in Artikel 3 des Grundgesetzes der Bundesrepublik Deutschland heißt es etwa: „Niemand darf wegen seines Geschlechts, seiner Abstammung, seiner Rasse, seiner Sprache, seiner Heimat und Herkunft, seines Glaubens, seiner religiösen oder politischen Anschauungen benachteiligt oder bevorzugt werden. Niemand darf wegen seiner Behinderung benachteiligt werden." Im Falle der genannten Chinesin, der wegen ihrer Herkunft die Anmietung einer Wohnung verweigert wird, ist dieses Gebot offensichtlich verletzt. Bei genauerem Hinsehen zeigt sich jedoch,

1 https://www.antidiskriminierungsstelle.de/SharedDocs/Pressemitteilungen/DE/2020/20200212 Coronavirus.html, 17. April 2020.

2 https://www.fr.de/panorama/corona-diskriminierung-alte-menschen-senioren-forscher-generationenkonflikt-zr-13654603.html, 17. April 2020.

https://www.djb.de/verein/Kom-u-AS/K4/pm20-15/, 21. April 2020. Diskriminierung ist allerdings kein spezifisches Kennzeichen krisenhafter Situationen, sondern allem Anschein nach ein Alltagsphänomen. So gab einer Umfrage von 2015 zufolge ein gutes Drittel der Befragten in Deutschland an, in den letzten zwei Jahren Opfer von Diskriminierung aufgrund von Geschlecht, ethnischer Herkunft oder sexueller Orientierung geworden zu sein. https:// www.antidiskriminierungsstelle.de/SharedDocs/Downloads/ DE/projekte/ Handout Umfrage Diskriminierung_in_Dtschl_2015.pdf?_blob=publicationFile\&v=1, 17. April 2020. 
dass es keineswegs so klar ist, was genau eine moralisch problematische Diskriminierung ausmacht. So gibt es beispielsweise zahlreiche Benachteiligungen auf der Basis von Gruppenmerkmalen, die wir gemeinhin nicht als problematisch erachten. Genannt seien die Regelungen, dass Jugendliche unter 16 keinen Alkohol trinken oder blinde Personen nicht Pilot*innen werden dürfen. Daneben gibt es Fälle, die schwieriger zu beurteilen sind: Stellt es eine unzulässige Diskriminierung dar, wenn ein Frauenhaus nur weibliche Psychologinnen einstellt? Und könnte das Alter der Patient*innen im Falle einer nötigen Beatmung nicht sogar ein legitimes Priorisierungskriterium darstellen? (Vgl. Kasmann 2020.)

Diese kurze Betrachtung zeigt, dass es keineswegs trivial ist festzustellen, was genau „Diskriminierung“ eigentlich ist, was mithin die Fälle problematischer von den Beispielen unproblematischer Ungleichbehandlungen unterscheidet. Erstaunlicherweise hat sich die philosophische Fachdebatte, im Unterschied etwa zur juristischen oder sozialpsychologischen, erst vor relativ kurzer Zeit diesem Gegenstand angenommen, wobei insbesondere die Arbeiten von Kasper Lippert-Rasmussen hervorzuheben sind.${ }^{4}$ Dies ist insofern zu begrüßen, als das Thema Diskriminierung nicht nur von hoher gesellschaftlicher Relevanz ist - man denke an die \#Metoo-Bewegung sowie den erstarkenden Rassismus angesichts von Zuwanderung -, sondern auch zahlreiche grundlagentheoretische philosophische Fragen berührt und spezifische begriffliche und normative Probleme aufwirft. So gilt es beispielsweise nicht nur, den Begriff der Diskriminierung zu schärfen, verschiedene Formen der Diskriminierung (z. B. direkt, indirekt, strukturell, institutionell) voneinander abzugrenzen und dabei zu klären, welche Gruppen überhaupt Opfer von Diskriminierung sein können, sondern auch zu spezifizieren, was eigentlich das moralische Übel diskriminierender Handlungen ist. Darüber hinaus gilt es zu klären, was die Diskriminierung verschiedener Gruppen und an verschiedenen „Schauplätzen“, wie Bildung oder Arbeitsmarkt, ausmacht, und es stellt sich die Frage, wie Diskriminierung auf gerechte Art und Weise effektiv unterbunden werden kann. ${ }^{5}$

4 Siehe Lippert-Rasmussen (2006; 2007; 2009; 2011; 2012; 2014) und, für einen umfassenden Überblick über die aktuelle Debatte, die Beiträge im Routledge Handbook of the Ethics of Discrimination, herausgegeben von Lippert-Rasmussen (2017).

5 Diese Aufzählung ist angelehnt an die Systematisierung von Lippert-Rasmussen (2017a, 2-15). 
Die Beiträge in diesem Schwerpunkt behandeln Themen aus all diesen Bereichen. Bevor ich die Aufsätze im Einzelnen vorstelle (Abschnitt 3), möchte ich einige Kontroversen und Themen skizzieren, die in der aktuellen philosophischen Diskussion um Diskriminierung eine zentrale Rolle spielen (Abschnitt 2). Dies dient nicht nur dem Überblick über relevante Teile des Diskurses, sondern erlaubt zudem eine Verortung der Beiträge aus diesem Schwerpunkt.

\section{Zentrale Themen und Kontroversen in der aktuellen Debatte um Diskriminierung}

\section{(i) Generischer oder normativer Diskriminierungsbegriff}

Die Differenzierung der Frage danach, was Diskriminierung sei, einerseits sowie der Analyse des spezifischen moralischen Übels der Diskriminierung andererseits mag zunächst verwundern; ist es doch im Deutschen eher unüblich, von einem normativ neutralen Diskriminierungsbegriff auszugehen. Wie die eingangs genannten Beispiele veranschaulichen, ist mit der Beschreibung eines Vorgangs als „diskriminierend“ normalerweise bereits eine negative moralische Wertung impliziert. Im angelsächsischen Sprachraum ist das anders und dementsprechend wird kontrovers über die Angemessenheit eines generischen oder eines normativen Diskriminierungsbegriffs diskutiert (Lippert-Rasmussen 2014, 15f., 24f.; Altman 2015; Thomsen 2017). Unter Zugrundelegung eines generischen Diskriminierungsbegriffs würden wir etwa sagen, dass die Tatsache, dass blinde Personen nicht Pilot*innen werden können, eine Diskriminierung darstellt, und in einem zweiten Schritt feststellen, dass sie moralisch in Ordnung ist. Ziehen wir einen normativen Begriff heran, würden wir diesen Sachverhalt gar nicht erst Diskriminierung nennen. Die Aussage „Diskriminierung ist falsch“ wäre eine reine Tautologie. Sofern nicht anders kenntlich gemacht, verwende ich im Folgenden einen normativen Diskriminierungsbegriff.

\section{(ii) Gruppenkriterium}

Bisher sind wir davon ausgegangen, dass das Vorliegen von Diskriminierung als notwendige Bedingung die ungerechtfertigte Benachteiligung von Individuen auf Basis eines Gruppenmerkmals umfasst. Aber um welche Merkmale handelt es sich hier eigentlich genau, was kennzeichnet mithin Eigenschaften wie die in Artikel 3 GG genannten, die gemeinhin als diskriminierungsre- 
levant, als „prohibited grounds“ (Moreau 2010, 143) gelten? Um diese Frage zu beantworten, muss eine überzeugende Definition von Diskriminierung ein sogenanntes Gruppenkriterium enthalten. Lippert-Rasmussen (2014, 15, 26) zufolge handelt es sich bei diskriminierungsrelevanten Merkmalen um solche, die die Zugehörigkeit zu einer sozial relevanten (socially salient) Gruppe anzeigen. Soziale Relevanz ist dadurch charakterisiert, dass die Zugehörigkeit zu dieser Gruppe soziale Interaktionen über verschiedene Kontexte hinweg beeinflusst (vgl. Lippert-Rasmussen 2014, 30). ${ }^{6}$ Dies trifft für die klassischen Diskriminierungsmerkmale wie race oder gender $\mathrm{zu}, 7$ schließt aber aus, dass Individuen aufgrund ihrer Augenfarbe, des Anfangsbuchstabens ihres Nachnamens oder weil sie Kleidung in sich beißenden Farben tragen, diskriminiert werden können (vgl. Scanlon 2008, 71). Andere Autor*innen halten diese Implikation für ungerechtfertigt und sprechen sich gegen ein Gruppenkriterium aus, da kein normativ relevanter Unterschied zwischen Benachteiligungen aufgrund verschiedener Gruppenmerkmale bestehe (vgl. Eidelson 2015, 26-30; Thomsen 2013, 2017).

\section{(iii) Was ist das spezifische moralische Übel der Diskriminierung?}

Die Frage nach dem spezifischen moralischen Übel fällt, wie gesagt, bei einem normativen Diskriminierungsbegriff mit der Frage danach, wie Diskriminierung zu definieren sei, zusammen und kann als Kern der Debatte gelten; entsprechend lang ist die Liste der bisher vorgeschlagenen wrong-making features diskriminierender Handlungen. So ist vorgebracht worden, Diskriminierung sei falsch, insofern sie an unveränderbare oder für die jeweilige Entscheidung irrelevante Merkmale anknüpfe oder auf fehlerhaften Stereotypen basiere. Allerdings sind weder alle gemeinhin als diskriminierungsrelevant angesehenen Merkmale unveränderbar (z. B. Religion) noch sind sie unbedingt irrelevant für die jeweilige Entscheidung, wie die Debatte um sogenannte reaction qualifications veranschaulicht (vgl. Mason 2017). Dabei

6 Für ähnliche Gruppenbestimmungen siehe Baber (2001, 53) und Young (1990, 46; 2001, 15).

$7 \quad$ Ich verwende hier und im Folgenden den englischsprachigen Begriff race und nicht „Rasse“, da die Begriffe auf einer ganz unterschiedlichen Geschichte basieren und unterschiedliche Konnotationen tragen. Auch die Bezeichnung people of color lasse ich unübersetzt in Ermangelung eines deutschsprachigen Pendants. Schließlich sei angemerkt, dass ich race und gender nicht als essentialistische biologische Kategorien, sondern als soziale Konstrukte betrachte, die deshalb aber nicht weniger real sind (vgl. etwa Haslanger 2012). 
handelt es sich um Qualifikationen, die sich durch die Reaktionen der Interaktionspartner*innen, etwa Kund*innen und Patient*innen, auf die betreffende Person ergeben. Höflichkeit und ein gepflegtes Äußeres können eine reaction qualification für eine Servicekraft in einem Café sein, Hautfarbe aber auch, wenn das Café in einem rassistisch geprägten Stadtteil liegt. Im letztgenannten Fall könnte der oder die Cafébesitzer*in argumentieren, dass Hautfarbe ein relevantes Merkmal bei der Auswahl der Servicekraft sei, insofern er oder sie bei Einstellung einer person of color ein Wegbleiben der Kund*innen zu befürchten hat. Dieses Beispiel zeigt darüber hinaus, dass Diskriminierung nicht notwendigerweise irrational ist. ${ }^{8}$ Auch müssen die Stereotype, auf denen Diskriminierung beruht, nicht falsch sein. Es ist korrekt, dass junge Frauen mit einer höheren Wahrscheinlichkeit (länger) Elternzeit nehmen als Männer; Geschlecht darf dennoch kein Einstellungsmerkmal sein.

Elaboriertere Ansätze argumentieren, Diskriminierung sei falsch, insofern oder wenn sie die Individuen ihrer deliberativen Freiheiten beraube (Moreau 2010), das Prinzip der Chancengleichheit verletze (Segall 2012) oder sie der diskriminierten Person Respekt verweigere und diese damit nicht als Individuum wertschätze (Eidelson 2015). ${ }^{9}$ Allerdings liefern auch diese Vorschläge keine notwendigen und hinreichenden Bedingungen für das Vorliegen von Diskriminierungen; so behandeln wir beispielsweise Menschen sehr häufig nicht als Individuum, sondern in ihrer jeweiligen Funktion (als Kassierer*in, Professor*in oder Polizist*in), und es kann auf allgemeinerer Ebene argumentiert werden, dass Stereotypisierung ein zentraler Mechanismus unseres Denkens ist, ohne den wir kaum mit anderen interagieren könnten (vgl. Schauer 2003, 75f.; 2017, 5of.; Anderson 2010, 45). Andersherum lassen sich jeweils Fälle von Diskriminierung angeben, die von den Ansätzen jeweils nicht erfasst werden können. Bei Chancengleichheit betrifft dies etwa Ungleichbehandlungen, die auf sozialisationsbedingte Unterschiede in den Lebensplänen und Ambitionen von Frauen und Männern zurückzuführen sind (vgl. Knight 2017, 145).${ }^{10} \mathrm{Zudem}$ ist dieser Ansatz

8 Zur Zurückweisung des Irrelevanz-Arguments siehe Hellman (2008, 114-137) und für eine Diskussion Halldenius (2017).

9 Für Übersichten siehe etwa Altman (2015) und Thomsen (2013; 2017).

10 Für eine Diskussion, inwiefern derartige Ungleichheiten überhaupt als Diskriminierung begriffen werden können und sollen, siehe Schouten (2017). 
mit dem Problem der Angleichung nach unten (levelling down) konfrontiert (vgl. Knight 2017, 145f.).

Aktuell werden in der Debatte insbesondere zwei Ansätze diskutiert: der konsequentialistisch schadensbasierte Ansatz von Lippert-Rasmussen (2014, 165-183) sowie die Idee Deborah Hellmans (2008), Diskriminierung sei falsch, insofern sie die diskriminierten Personen herabsetze. ${ }^{11}$ Auch diese Vorschläge sind nicht ohne Kritik geblieben; so wird insbesondere in Frage gestellt, ob Diskriminierung immer einen Schaden auf Seiten der Diskriminierten impliziere (vgl. Horta 2015; Slavny und Parr 2016), während wiederum Hellmans Definition von Diskriminierung als Herabwürdigung als zu stark kritisiert worden ist, da sie bestimmten Personen den gleichen moralischen Wert oder gar ihre Menschlichkeit abspreche (vgl. Arneson 2013, 9194; Lippert-Rasmussen 2014, 134-139). Nichtsdestotrotz bringen beide Autor*innen bestimmte Intuitionen auf den Punkt: Diskriminierung, so scheint es, hat etwas mit Benachteiligung bzw. mit Einbußen an Wohlergehen, aber auch mit sozialen Bedeutungen und sozialer Hierarchie zu tun. Schließlich kann die Suche nach dem einen wrong-making feature, das allen Arten der Diskriminierung zugrunde liegt, selbst kritisiert werden (vgl. Blum 2013). Womöglich sind die Phänomene, die gemeinhin mit dem Begriff der Diskriminierung bezeichnet werden, derart disparat, dass nur ein pluralistischer Ansatz erfolgversprechend ist.

\section{(iv) Verschiedene Arten der Diskriminierung I: Intentionen, Strukturen und implizite Vorurteile}

Öffentliche oder private Debatten um Diskriminierung, insbesondere um rassistische oder sexistische, sind häufig emotional sehr aufgeladen. Während eine Seite der anderen Diskriminierung vorwirft, weist diese das brüskiert von sich. Dies dürfte sehr häufig mit unterschiedlichen Begriffsverwendungen und Assoziationen, insbesondere im Hinblick auf die Absichten und den Charakter des oder der Handelnden, zu tun haben. So denken viele vermutlich bei rassistischer Diskriminierung an eine*n „klassische* $n$ “ Rassist*in, der oder die eine negative und abwertende Einstellung gegenüber people of color hat und diese Personen bewusst und absichtlich benachteiligt. Jemanden der rassistischen Diskriminierung zu bezichtigen, ist diesem Begriffsverständnis zufolge ein schwerwiegender moralischer Vorwurf (vgl.

11 Ähnlich wie Hellman argumentiert Scanlon (2008). Kritik an Scanlon übt Lippert-Rasmussen (2012 und 2014, 139-152). 
Blum 2002, 34). ${ }^{12}$ Während es derartige Fälle offener Diskriminierung sicherlich gibt, sind sie im öffentlichen und privatwirtschaftlichen Raum zumindest rechtlich verboten und im Privaten generell geächtet. Damit sind Vorurteile und Benachteiligungen indes nicht verschwunden, sondern haben andere Formen angenommen.

So haben vergangene (offene) Diskriminierungen zum einen gesellschaftliche Strukturen und Institutionen geschaffen, die zu Benachteiligungen marginalisierter Gruppen führen können, obwohl dies niemand beabsichtigt. Werden beispielsweise freie Stellen in einem Unternehmen oder einer Organisation über Empfehlungen der aktuellen Mitarbeiter*innen besetzt und finden sich unter dem Mitarbeiter*innenstab aufgrund früherer Zugangsbeschränkungen keine Frauen, people of color oder behinderte Personen, so setzt diese per se harmlose Regelung Diskriminierung fort (vgl. Wasserman 1998, 811; Blum 2002, 23). Fälle wie diese werden häufig als institutionelle Diskriminierung bezeichnet (vgl. ebd.). Wird auch der Effekt informellerer gesellschaftlicher Strukturen, wie Normen, soziale Praktiken und Narrative, mitberücksichtigt, spricht man von struktureller Diskriminierung (vgl. Mikkola 2017, 289).13

Zum anderen hat die sozialpsychologische Forschung gezeigt, dass Benachteiligungen von Mitgliedern marginalisierter Gruppen aufgrund unbewusster Vorurteile und Stereotype (fort)bestehen. ${ }^{14}$ Ein bekanntes Beispiel

12 Das ist der Grund, warum einige Autor*innen den Begriff des Rassismus an entsprechende negative Einstellungen knüpfen wollen. So schreibt etwa Anderson (2010, 48): „let us reserve ,racism‘ for judgments of serious vice, while observing that not all injustice is caused by a vicious character" und für Arthur $(2007,17)$ besteht Rassismus in ,an attitude of hostility or indifference“. Siehe auch Blum 2002, 2, 9. Young $(1990,196)$ plädiert dafür, auch den Diskriminierungsbegriff entsprechend eng zu führen: „The concept of discrimination [...] should be restricted to the explicit exclusion or preference of some people in the distribution of benefits, the treatment they receive, or the positions they occupy, on account of their social group membership."

13 Zum Begriff der gesellschaftlichen Struktur und der strukturellen Ungleichheit siehe Young (2001, 9-15).

14 Als Stereotype werden kognitive Schemata über Klassen von Objekten bezeichnet, die bei der Wahrnehmung von und der Interaktion mit einzelnen Objekten dieser Klasse helfen (vgl. Anderson 2010, 45). In Bezug auf Personengruppen lassen sich Stereotype mit Dovidio et al. $(2010,8)$ definieren als „associations and beliefs about the characteristics and attributes of a group and its members that shape how people think about and respond to the 
ist der sogenannte Shooter-Bias-Test, in dem Versuchspersonen in einer Art Videospiel mit bewaffneten und unbewaffneten Personen unterschiedlicher Hautfarbe konfrontiert werden und schnell entscheiden müssen, ob sie schieBen oder nicht (vgl. Kelly und Roedder 2008, 526; Jost et al. 2009, 50f.; Holroyd 2017, 385f.). Im Ergebnis schießen die Versuchspersonen häufiger und machen mehr Fehler in ihrer Gefahreneinschätzung, wenn die Zielperson eine person of color ist. Ebenfalls gut belegt ist die Beobachtung, dass Frauen in klassischen Männerberufen bei gleicher Leistung oft als weniger kompetent beurteilt werden als Männer (vgl. Valian 2005). ${ }^{15}$ Eine Studie unter BWL- und VWL-Studierenden konnte zudem nachweisen, dass weibliche Lehrkräfte von Studierenden systematisch schlechter evaluiert worden sind als männliche (vgl. Mengel et al. 2019). Selbst identisches Lehrmaterial schnitt schlechter in der Bewertung ab, wenn die Lehrperson eine Frau war. Dabei kann den Versuchspersonen nicht durchweg bewusst rassistische oder sexistische Diskriminierung vorgeworfen werden. Vielmehr zeigt sich, dass auch Personen, die davon überzeugt sind, keinerlei explizite Vorurteile gegenüber der jeweiligen marginalisierten Gruppe zu hegen, durchaus implizite Vorurteile haben und entsprechend handeln können (vgl. Kelly und Roedder 2008; Jost et al. 2009, 46). ${ }^{16}$ Das diskriminierende Verhalten geschieht hier also unbewusst.

Insofern ein Großteil an Strukturen rassistisch und sexistisch geprägt sein dürfte, um bei diesen Beispielen zu bleiben, und angesichts der Tatsache, dass implizite Vorurteile weit verbreitet sind, müssen diesem Begriffsverständnis nach wesentlich mehr Handlungen als rassistisch oder sexistisch diskriminierend gelten. ${ }^{17}$ Die Frage der Verantwortungszuschreibung

group“. „Vorurteile“ verstehe ich demgegenüber als affektiv aufgeladene Stereotype. Besonders problematisch sind sie, wenn das affektive Festhalten des Individuums an Vorurteilen mit einer Weigerung einhergeht, sie im Lichte von gegenläufigen Beispielen zu verändern (vgl. Fricker 2007, 35).

$15 \mathrm{Zu}$ unbeabsichtigter Diskriminierung bei der Einstellung von Personen siehe auch Uhlmann und Cohen (2005).

16 Siehe zum Impliziten Assoziationstest, der am häufigsten verwendeten Methode zur Erhebung impliziter Stereotype und Vorurteile, Kelly und Roedder (2008, 524-526), Jost et al. (2009, 45f.) sowie Brownstein (2019, Abschnitt 1.2).

17 Dementsprechend würden etwa Haslanger (2012) oder DiAngelo (2018) den Begriff des Rassismus ausweiten. So schreibt Haslanger (2012, 335): „If we want the term ,racism' to capture all the barriers to racial justice, I submit that it is reasonable to count as ,racist' not only the attitudes and actions of indi- 
und der Schuldhaftigkeit der Akteure ist in diesen Fällen indes keineswegs so klar, wie es bei der „klassischen“, expliziten Diskriminierung der Fall ist. ${ }^{18}$ Jedenfalls impliziert ein Diskriminierungsbegriff, der auch die zuletzt diskutierten Phänomene einfängt, nicht mehr die starke moralische Verurteilung der handelnden Person, wie wir sie oben konstatiert hatten, und damit wird zumindest ein Grund deutlich, warum der öffentliche Diskriminierungsdiskurs oft so emotional verläuft: die verschiedenen Parteien mögen unterschiedliche Diskriminierungsbegriffe mit unterschiedlichen Implikationen für die moralische Bewertung des Charakters und die Schuldhaftigkeit der handelnden Person im Sinn haben.

\section{(v) Verschiedene Arten der Diskriminierung II: direkte, indirekte und statistische Diskriminierung}

Im vorherigen Abschnitt wurde deutlich, dass zum Teil sehr unterschiedliche Phänomene mit dem Begriff der Diskriminierung bezeichnet werden. Tatsächlich ergibt die bisher wohl umfangreichste Taxonomie bei Lippert-Rasmussen (2014, 45) 64 verschiedene Arten der Diskriminierung. An dieser Stelle und mit Hinblick auf die Beiträge in diesem Schwerpunkt reicht es aus, zwei weitere zentrale Unterscheidungen zu nennen: die zwischen direkter und indirekter Diskriminierung einerseits sowie die zwischen statistischer und nichtstatistischer Diskriminierung andererseits. Die erstgenannte Differenzierung ist keineswegs eindeutig, wird durch unterschiedliche Begriffsverwendungen in verschiedenen Rechtssystemen erschwert und manche Autoren bezweifeln sogar, dass indirekte Diskriminierung überhaupt eine sinnvolle Kategorie sei (vgl. Eidelson 2015). Als eine grobe Annäherung lässt sich sagen, dass direkte Diskriminierung eine unmittelbar an das diskriminierungsrelevante Merkmal anknüpfende Ungleichbehandlung ist (disparate treatment), während indirekte Diskriminierung zwar ein neutrales Unterscheidungskriterium anwendet, aber einen unverhältnismäBigen negativen Effekt auf eine bestimmte Personengruppe bewirkt (disparate impact). Um auf die eingangs genannten Beispiel zurückzukommen:

viduals but the full range of practices, institutions, policies, and suchlike that, I've argued, count as racially oppressive."

18 Siehe dazu Holroyd (2012), Brownstein (2019, Abschnitt 4.1) sowie die Beiträge in Brownstein und Saul (2016, Teil 1). Fruchtbar scheinen hier auch Frickers (2007, 98-108) Überlegungen zu vergangenem Unrecht sowie die Unterscheidung zwischen Schuldzuweisung und moralischer Enttäuschung. 
Das Beispiel der chinesischen Studentin ist ein Fall direkter Diskriminierung, während die Diskriminierung von Frauen beim Kurzarbeitergeld indirekt erfolgt, insofern die Maßnahme nicht an das Merkmal Geschlecht, sondern an Nettolohn und Steuerklasse anknüpft, was die Gruppe der Frauen aber im Vergleich zur Gruppe der Männern insgesamt schlechterstellt. Die begrifflichen und normativen Probleme, die diese Unterscheidung mit sich bringt, sind Gegenstand anhaltender Diskussionen (vgl. Wasserman 1998; Selmi 2013; Lippert-Rasmussen 2014, 54-78; Altman 2015; Khaitan 2017; Thomsen 2017).

Statistische Diskriminierung wiederum unterscheidet sich von nichtstatistischer dadurch, dass das Vorliegen eines diskriminierungsrelevanten Merkmals hier als ein Indikator für das die diskriminierende Person eigentlich interessierende Merkmal fungiert (vgl. Schauer 2017, 43). Stellt ein Arbeitgeber keine jungen Frauen ein, weil er davon ausgeht, dass diese aufgrund von Elternzeit als Arbeitskräfte ausfallen werden, und nicht, weil er Frauen hasst, dann ist das ein Fall von statistischer Diskriminierung. Dieses Beispiel deutet bereits an, wo das Problem dieses Konzepts liegt: Insofern Diskriminierung häufig auf bestimmten Stereotypen, also auf Verallgemeinerungen, beruht, ist sie immer schon statistisch (vgl. Lippert-Rasmussen 2014, 82f.). Dass jemand bestimmte Personengruppen für minderwertig hält, ohne dabei irgendwelche Assoziationen über ihren Charakter und ihr Verhalten zu haben, ist schwer vorstellbar. ${ }^{19}$ Andersherum wird etwa am Beispiel der Nichteinstellung junger Frauen nochmals deutlich, dass (statistische) Diskriminierung keiner ablehnenden Haltung der Diskriminierenden gegenüber den Diskriminierten bedarf.

\section{(vi) Sphäre der Diskriminierung}

Im Kontext der statistischen Diskriminierung ist die Praxis des (racial) profiling besonders umstritten (vgl. Schauer 2003, 155-198; 2017, 50f.; Lippert-Rasmussen 2014, 272-301). Dies dürfte nicht zuletzt auch daran liegen, dass es hier der Staat ist, der seine Bürger*innen auf Basis eines diskriminierungsrelevanten Merkmals ungleich behandelt; schließlich verbieten viele Verfassungen, darunter das eingangs zitierte Deutsche Grundgesetz, derartige Diskriminierung eindeutig (vgl. Collins 2017, 361). Ist es doch der Staat,

19 Zur Problematik der Abgrenzung von statistischer und nichtstatistischer Diskriminierung sowie zur moralischen Problematik letzterer siehe Schauer (2003; 2017) und Lippert-Rasmussen (2014, 79-97). 
der die Freiheiten und Rechte der Individuen definiert und durchsetzt und somit eine erhebliche Macht über die Vorenthaltung von Chancen, Respekt und Anerkennung - oder wie auch immer das moralische Übel von Diskriminierung bestimmt wird - hat. Eine weitere Sphäre der Diskriminierung stellt der teils öffentlich, teils privat organisierte Zugang zu Bildung, Wohnraum und Arbeit dar. ${ }^{20}$ Wesentlich strittiger ist die Frage, inwiefern der persönliche Nahbereich ebenfalls als eine Sphäre möglicher Diskriminierung angesehen werden sollte - die Frage nach der rechtlichen Regulierung derartiger Diskriminierungen einmal beiseitegelegt (vgl. Collins 2017). Wenn es falsch ist, eine Person aufgrund ihrer Religion nicht einzustellen, wäre es dann nicht ebenso falsch, sie als einzige unter den Kolleg*innen nicht zur privaten Geburtstagsfeier einzuladen? Und sollte es nicht analog als diskriminierend gegenüber persons of color gelten, ausschließlich weiße Personen als Sexualpartner*innen in Erwägung zu ziehen? (Vgl. Liu 2015; Lazenby und Butterfield 2017; Fourie 2017.)

Diese Fragen haben in der Literatur bislang noch wenig Beachtung gefunden. Sie mögen auf den ersten Blick auch befremdlich erscheinen, schließlich scheint es eine rein persönliche Angelegenheit zu sein, wen man zu seiner Geburtstagsfeier einlädt und wen nicht, wen man attraktiv findet und wen nicht. Allerdings ist es naheliegend anzunehmen, dass auch Sympathie und Anziehung von Vorurteilen beeinflusst werden. ${ }^{21}$ Insofern einiges darauf hindeutet, dass regelmäßige persönliche Kontakte mit Mitgliedern marginalisierter Gruppen unter bestimmten Bedingungen zum Abbau von Vorurteilen führen können (vgl. Kang und Banaji 2006, 1101-1109; Anderson 2010, 123-127; Liu 2015, 257f.), können Beziehungen im persönlichen Nahbereich zumindest nicht per se der moralischen Bewertung entzogen werden. Inwiefern hier von Diskriminierung die Rede sein sollte und wie, im Falle einer negativen Antwort auf diese Frage, die angemessene „Sphäre“ von Diskriminierung bestimmt werden kann, bleibt zu diskutieren.

20 Siehe dazu etwa die Gleichbehandlungsrichtlinien der Europäischen Union: https://www.antidiskriminierungsstelle.de/DE/ThemenUndForschung/ Recht und gesetz/EU-Richtlinien/eu-Richtlinien node.html, 25 April 2020. racial group unattractive is often a combination of lack of positive portraits of them in society and our own failure to allow them into our personal lives as equals." 


\section{(vii) Antidiskriminierungsmaßnahmen - umgekehrte Diskriminierung?}

Ganz im Gegensatz zur Diskriminierung im persönlichen Nahbereich sind Antidiskriminierungsmaßnahmen - oder, allgemeiner, Affirmative Action (AA) - insbesondere in den USA Gegenstand einer umfangreichen Debatte. ${ }^{22} \mathrm{AA}$ bezeichnet Maßnahmen, die darauf abzielen, die Repräsentation bislang unterrepräsentierter Gruppen im Bereich Bildung, Beschäftigung und Kultur zu erhöhen (vgl. Fullinwider 2018). Dazu zählen insbesondere Quotenregelungen sowie die Praxis der bevorzugten Anstellung bzw. Zulassung von Mitgliedern einer unterrepräsentierten Gruppe bei gleicher Eignung. AA ist insofern ein weiterer Begriff als Antidiskriminierung, als er verschiedene Begründungen der Maßnahmen zulässt; neben dem Ziel, Diskriminierung entgegenzuwirken, kann AA etwa der Kompensation für vergangenes Unrecht, der Förderung epistemischer Diversität oder einer umfangreicheren gesellschaftlichen Integration dienen (vgl. Anderson 2010, 135-154). ${ }^{23}$ Ich konzentriere mich an dieser Stelle auf das Ziel, Diskriminierung zu unterbinden bzw. zu verhindern.

Hinsichtlich der Argumente, die gegen die Notwendigkeit und die Gerechtigkeit von AA-Maßnahmen mit dem Ziel der Antidiskriminierung, gerade in Form der bevorzugten Anstellung, vorgebracht werden, möchte ich zwei nennen, die einen unmittelbaren Bezug zum hier interessierenden Thema aufweisen. So wird erstens argumentiert, dass die bloße unterschiedliche Repräsentation bestimmter Gruppen in bestimmten Berufen oder Positionen per se nicht den Schluss zulasse, dass hier eine Diskriminierung stattgefunden habe; ein geringer Anteil von Frauen in der akademischen Philosophie beispielsweise könne durch unterschiedliche Interessen oder Fähigkeiten erklärt werden (vgl. Sesardic und De Clercq 2014). ${ }^{24}$ AA sei somit gar nicht notwendig. Ungerecht seien entsprechende Maßnahmen zudem, zweitens, weil sie Mitglieder der jeweils dominanten Gruppe diskriminierten. Wenn etwa eine Universität angesichts des geringen Frauenanteils unter ihren wis-

22 Siehe für einen Überblick und weitere Referenzen Fullinwider (2018).

23 Für die Relevanz epistemischer Diversität in verschiedenen Lebensbereiche siehe kürzlich eindrucksvoll Criado-Perez (2019). Das Argument der Kompensation vergangenen Unrechts ist problematisch und scheint mittlerweile nur noch selten vertreten zu werden (vgl. ebd., 137-141; Lippert-Rasmussen 2017b).

Differenzierter dazu Lippert-Rasmussen (2009). 
senschaftlichen Mitarbeiter*innen beschließt, für einen bestimmten Zeitraum nur noch Frauen einzustellen, so sei dies diskriminierend gegenüber Männern. ${ }^{25}$

Eine Antwort auf den ersten Einwand kann offenbar nicht allein auf der Basis begrifflicher und moralphilosophischer Überlegungen, sondern nur empirisch informiert erfolgen. Dabei gilt es insbesondere, nicht nur vergangene explizite, sondern auch andauernde strukturelle Diskriminierung sowie auf unbewussten sozialpsychologischen Mechanismen basierende Ausgrenzungen bestimmter Gruppen zu berücksichtigen (vgl. Kang und Banaji 2006). Neben den unter (iv) bereits genannten impliziten Vorurteilen ist hier insbesondere die Bevorzugung der eigenen Gruppe (ingroup bias oder ingroup favoritism) zu nennen (vgl. Dasgupta 2004). Mit der Anerkennung derartiger Mechanismen verschiebt sich die AA-Debatte weg von dem rückwärtsgewandten Argument der Kompensation vergangenen Unrechts, hin zur gegenwarts- und zukunftsorientierten Behebung von aktueller Diskriminierung (vgl. ebd.). Dass diese vorliegt, bleibt natürlich jeweils im Einzelfall zu überprüfen und es ist ebenfalls eine empirische Frage, inwiefern bestimmte AA-Maßnahmen wirksam Abhilfe schaffen können. Inwiefern wiederum die Kritik zutrifft, dass AA Mitglieder der dominanten Gruppe „diskriminiert“, hängt vom zugrunde gelegten Diskriminierungsbegriff ab, setzt also eine Auseinandersetzung mit einigen der in diesem Beitrag skizzierten Fragen voraus. ${ }^{26}$

25 Dies ist kein hypothetisches Beispiel. Die Technische Universität Eindhoven (Niederlande) hat im Sommer 2019 verkündet, die Stellenausschreibungen in den darauffolgenden zwei Jahren zunächst nur für Frauen zu öffnen: https:// www.dutchnews.nl/news/2019/06/eindhoven-university-opens-academic-jobs-to-women-only/, 25. April 2020. Dass Männer in der Anstellungspraxis der akademischen Philosophie diskriminiert werden, behaupten etwa Sesardic und De Clercq (2014). Siehe zur Entkräftung dieses Arguments Rössler 2012, 374f.

26 Für Young (1990, 193-198) stellt AA Diskriminierung dar, ist aber moralisch gerechtfertigt. Suk (2017) hingegen argumentiert, dass AA die zentralen Eigenschaften von Diskriminierung nicht erfüllt und somit auch nicht unter diesen Begriff zu subsummieren sei. Daraus, dass AA-Maßnahmen keine Diskriminierung darstellen, folgt allerdings nicht, dass sie moralisch legitim sind. 


\section{3. Überblick über die Beiträge des Schwerpunkts}

Vor dem Hintergrund dieses Überblicks über zentrale Themen und Kontroversen der aktuellen philosophischen Debatte um Diskriminierung gilt es nun, die Beiträge in diesem Schwerpunkt vorzustellen und in die diskutierten Themenfelder einzuordnen.

Der Beitrag von Hauke Behrendt, Diskriminierung und das Kriterium der Gruppenzugehörigkeit, befasst sich mit dem normativen Begriff der direkten Diskriminierung (vgl. (i)), argumentiert für die Relevanz eines Gruppenkriteriums (vgl. (ii)) und schlägt eine spezifische Deutung des Gruppenbegriffs vor, die zugleich etwas über die Art der mit Diskriminierung verbundenen Ungerechtigkeit aussagt (vgl. (iii)). Im Zuge der Argumentation für die Notwendigkeit eines Gruppenkriteriums für einen überzeugenden Diskriminierungsbegriff weist Behrendt einen an beliebigen Merkmalen ansetzenden Diskriminierungsbegriff als zu weit und auch die Relevanzbedingung als unplausibel zurück (vgl. (iii)). Hinsichtlich möglicher Deutungen des Gruppenbegriffs differenziert Behrendt eine realistische und eine klassifikatorische Lesart und zeigt auf, dass nur die klassifikatorische Interpretation überzeugt. Damit kommt er zu dem glücksegalitaristisch anmutenden Fazit, dass „komparative Benachteiligungen auf der Basis sozial bedeutsamer Klassifikationen moralisch nur dann zulässig [sind], wenn sie die Folge von Umständen sind, die die Person selbst zu verantworten hat". Dies sei bei sozialer Artzugehörigkeit (race, gender etc.) niemals der Fall.

Michael Oliva Córdoba setzt in dem Beitrag Diskriminierung und Verwerflichkeit: Huxleys Albtraum und die Rolle des Staates weniger am Konzept, sondern eher an der Sphäre der Diskriminierung an und argumentiert, dass Diskriminierung primär ein Problem im Bereich des Politischen sei (vgl. (vi)). Das Politische bestimmt er dabei in Anlehnung an Thomas Hobbes als das Verhältnis vom Staat zu den Bürger*innen; ausgeklammert bleibt damit die Interaktion zwischen Bürger*innen. Einen deskriptiven Diskriminierungsbegriff verwendend (vgl. (i)) lautet Córdobas These, dass Diskriminierung durch den Staat immer unzulässig sei, da es sich dabei um Machtmissbrauch handele, im Verhältnis zwischen den Bürger*innen hingegen nicht unbedingt, wie er anhand der Beispiele der Partner*innenwahl und des ökonomischen Tausches am Markt illustriert. Auf diskriminierenden Präferenzen basierende Entscheidungen seien hier erlaubt, da es sich um eine Beziehung unter Gleichen handele. Huxleys - und wohl auch Córdobas Albtraum besteht in der Aufhebung dieses privaten Raums durch staatliche 
Einmischung (vgl. (vi)). AA-Maßnahmen sieht der Autor dabei bereits als Schritt in die falsche Richtung an (vgl. (vii)).

Während sich die Beiträge von Behrendt und Córdoba auf einer relativ abstrakten Ebene mit direkter Diskriminierung auseinandersetzen, haben die übrigen vier Beiträge jeweils einen spezifischeren Anwendungsbereich zum Gegenstand. So setzten sich Regina Schidel und Heiner Koch mit zwei aktuellen Themen aus dem Bereich der angewandten Ethik auseinander, Christine Bratu und Anna Leuschner diskutieren AA-Maßnahmen zugunsten von Frauen im Kontext der akademischen Philosophie. Schidel befasst sich in ihrem Beitrag Pränataldiagnostik als Instanz von struktureller Diskriminierung? Überlegungen zur Debatte um den PraenaTest und seine Auswirkungen auf Menschen mit Behinderung mit der Diskriminierung von Menschen mit Trisomie 21. Der sogenannte PraenaTest ist ein vorgeburtlicher Bluttest, der die Diagnose des Down-Syndroms erlaubt und seit 2019 in bestimmten Fällen von der gesetzlichen Krankenversicherung finanziert wird. Schidel wirft die Fragen auf, inwiefern der Test, dem im Falle der Diagnose von Trisomie 21 häufig eine Abtreibung folgt, Menschen mit Down-Syndrom diskriminiert und inwiefern die Aufnahme des Tests in den Leistungskatalog der gesetzlichen Krankenversicherung diese Diskriminierung verstärkt. Um diese Fragen zu beantworten, diskutiert sie die Diskriminierungsbegriffe von Lippert-Rasmussen und Hellman (vgl. (iii)) und argumentiert unter Rückgriff auf Foucaults Begriff der Biomacht, dass diese Konzepte strukturell zu erweitern seien (vgl. (iv)), um das hier betrachtete Phänomen einfangen zu können. Insofern die Etablierung des PraenaTests eine strukturelle Diskriminierung von Menschen mit Trisomie 21 bewirke, so Schidels Fazit, solle seine Anwendung strikt auf bestimmte Risikofälle beschränkt werden.

Koch betrachtet in seinem Beitrag Intransparente Diskriminierung durch maschinelles Lernen Diskriminierungen durch Algorithmen und zeigt auf, dass dieses Phänomen mit besonderen Herausforderungen für die philosophische Diskriminierungsdebatte verknüpft ist. Im Unterschied zu den anderen Beiträgen geht es bei Koch primär um statistische Diskriminierung (vgl. (v)), wobei er einen normativen Diskriminierungsbegriff zugrunde legt (vgl. (i)). Während man jedoch statistische Diskriminierung durch Algorithmen auf der Basis von klassischerweise als diskriminierungsrelevant geltenden Merkmalen noch relativ gut entdecken könne und dieses Problem in der Literatur auch bereits ausführlich diskutiert worden sei, stellt Koch heraus, dass man bei selbst lernenden Algorithmen häufig gar nicht wisse, anhand welcher Merkmale diese zwischen Personen differenzierten. Diese Merk- 
male können zudem für Menschen unverständlich sein. Koch argumentiert, dass man hier dennoch von Diskriminierung sprechen könne, indem er die Notwendigkeit eines Gruppenkriteriums zurückweist (vgl. (ii)) und erörtert, dass Diskriminierung keiner mentalen Eigenschaften auf Seiten der diskriminierenden Instanz bedürfe (vgl. (iv), (v)). Insofern Algorithmen diskriminieren können, es aber angesichts ihrer Intransparenz erhebliche Schwierigkeiten gibt, dies festzustellen und ggf. zu beheben, plädiert Koch dafür, in bestimmten Kontexten nur Algorithmen zu verwenden, bei denen diese Probleme überwunden werden können.

Bratu befasst sich in dem Text Ist die Praxis bevorzugter Anstellung moralisch zulässig? mit der Frage, ob die bevorzugte Anstellung von Frauen in der akademischen Philosophie auch dann zulässig sei, wenn dadurch vom Prinzip der Bestenauswahl abgewichen würde (vgl. (vii)). Als der oder die beste Bewerber*in gilt Bratu zufolge dabei die Person mit dem größten akademischen Potential. Um die These zu stützen, dass die Praxis der bevorzugten Anstellung moralisch zulässig ist und gegenüber Mitbewerbern keine Diskriminierung in Form der Vorenthaltung von Chancengleichheit darstellt (vgl. (iii)), rekonstruiert die Autorin die Argumentation der sogenannten traditionellen (man könnte auch sagen: meritokratischen) Sichtweise, zeigt auf, dass diese modifiziert werden muss, um zu überzeugen, und in der abgeschwächten Version grundsätzlich mit der Praxis der bevorzugten Anstellung vereinbar ist. Berücksichtige man zudem, dass aktuell implizite Vorurteile und andere sozialpsychologische Mechanismen der Chancengleichheit in der akademischen Philosophie entgegenstünden (vgl. (iv) und (vii)), dann seien die Ziele der traditionellen Sichtweise mithilfe der diskutierten Maßnahme sogar besser zu erreichen.

Leuschner schließlich untersucht die Legitimität von AA-Maßnahmen innerhalb der akademischen Philosophie nicht auf der Ebene der Anstellung, sondern auf der Ebene der Planung philosophischer Fachtagungen. Unter dem Titel Quoten für Hauptvorträge? Moralische, soziale und epistemische Aspekte akademischer Quotenregelungen am Beispiel der Gendered Conference Campaign verteidigt sie diese Kampagne insbesondere gegen einige von David Benatar vorgebrachte Einwände (vgl. (vii)). Leuschner argumentiert, dass eine größere Repräsentation von Frauen als Hauptrednerinnen auf Konferenzen nicht nur moralisch gefordert, sondern auch unter epistemischen Gesichtspunkten sinnvoll ist.

Insgesamt decken die Beiträge in diesem Schwerpunkt damit ein breites Spektrum der in der Diskriminierungsdebatte diskutierten Themen ab, 
positionieren sich zu einzelnen Fragen ganz unterschiedlich und laden somit auch zu Widerspruch ein, sodass sie der deutschsprachigen Diskussion über Diskriminierung (hoffentlich) neue Impulse verleihen.

\section{Literatur}

Altman, Andrew. 2015. „Discrimination“. Stanford Encyclopedia of Philosophy, https://plato.stanford.edu/entries/discrimination/, 8. Juli 2017.

Anderson, Elizabeth. 2010. The Imperative of Integration. Princeton, Oxford: Princeton UP.

Arneson, Richard. 2013. „Discrimination, disparate impact, and theories of justice“. In Philosophical Foundations of Discrimination Law, hrsg. von Deborah Hellman und Sophia Moreau, 87-111. Oxford.

Arthur, John. 2007. Race, Equality, and the Burdens of History. Cambridge.

Baber, Harriet E. 2001. „Gender conscious“. Journal of Applied Philosophy 18(1), $53-63$.

Blum, Lawrence. 2002. "I'm not a Racist, But ..." The Moral Quandary of Race. Ithaca/London.

Blum, Lawrence. 2013. „Racial and other asymmetries. A problem for the protected category framework for anti-discrimination thought“. In Philosophical Foundations of Discrimination Law, hrsg. von Deborah Hellman und Sophia Moreau, 182-199. Oxford.

Brownstein, Michael. 2019. „Implicit biases“. Stanford Encyclopedia of Philosophy. https://plato.stanford.edu/entries/implicit-bias, 27. April 2020.

Brownstein, Michael, und Jennifer Saul, Hrsg. 2016. Implicit Bias and Philosophy, Volume 2: Moral Responsibility, Structural Injustice, and Ethics. Oxford.

Collins, Hugh. 2017. „Discrimination and the private sphere“. In The Routledge Handbook of the Ethics of Discrimination, hrsg. von Kasper Lippert-Rasmussen, 360-368. London.

Criado-Perez, Carolina. 2019. Invisible Women: Exposing Data Bias in a World designed for Men, London.

Dasgupta, Nilanjana. 2004. „Implicit ingroup favoritism, outgroup favoritism, and their behavioral manifestations“. Social Justice Research 17(2): 143-169.

DiAngelo, Robin. 2018. Why it is So Hard for White People to Talk about Racism. Beacon.

Dovidio, John F., Miles Hewstone, Peter Glick und Victoria M. Esses. 2010. „Prejudice, stereotyping and discrimination: theoretical and empirical overview". In The SAGE Handbook of Prejudice, Stereotyping and Discrimination, hrsg. von John F. Dovidio, Miles Hewstone, Peter Glick und Victoria M. Esses, 3-28. Los Angeles. 
Eidelson, Benjamin. 2015. Discrimination and Disrespect. Oxford.

Fourie, Carina. 2017. „Wrongful private discrimination and the egalitarian ethos“. In The Routledge Handbook of the Ethics of Discrimination, hrsg. von Kasper Lippert-Rasmussen, 421-432. London.

Fricker, Miranda. 2007. Epistemic Injustice. Power and the Ethics of Knowing. Oxford.

Fullinwider, Robert. 2018. „Affirmative action“. Stanford Encyclopedia of Philosophy, https://plato.stanford.edu/entries/affirmative-action/, 22. Oktober 2018.

Halldenius, Lena. 2017. „Discrimination and irrelevance“. In The Routledge Handbook of the Ethics of Discrimination, hrsg. von Kasper Lippert-Rasmussen, 108118. London.

Haslanger, Sally. 2012. Resisting Reality. Social Construction and Social Critique. Oxford.

Hellman, Deborah. 2008. When is Discrimination Wrong? Cambridge Mass.

Hellman, Deborah, und Sophia Moreau, Hrsg. 2013. Philosophical Foundations of Discrimination Law, Oxford.

Holroyd, Jules. 2012. „Responsibility for implicit biases“. Journal of Social Psychology 3(3), 274-306.

Holroyd, Jules. 2017. „The social psychology of discrimination“. In The Routledge Handbook of the Ethics of Discrimination, hrsg. von Kasper Lippert-Rasmussen, 381-393. London.

Horta, Oscar. 2015 „Does discrimination require disadvantage?“. Moral Philosophy and Politics 2(2), 277-297.

Jost, John T., Laurie A. Rudman, Irene V. Blair, Dana R. Carney, Nilanjana Dasgupta, Jack Glaser und Curtis D. Hardin. 2009. „The existence of implicit bias is beyond reasonable doubt: A refutation of ideological and methodological objections and executive summary of ten studies that no manager should ignore“. Research in Organizational Behavior 29: 39-69.

Kang, Jerry, und Mahzarin R. Banaji. 2006. „Fair measures: a behavioral realist revision of affirmative action“. California Law Review 1063(4), 1063-1118.

Kasmann, Tobias. 2020. Corona-Triage, Risikogruppen und Altersdiskriminierung, https://www.praefaktisch.de/covid-19/corona-triage-risikogruppen-und-altersdiskriminierung/, 21. April 2020.

Kelly, Daniel, und Erica Roedder. 2008. „Racial cognition and the ethics of implicit bias“. Philosophy Compass 3(3), 522-540.

Khaitan, Tarunabh. 2017. „Indirect discrimination“. In The Routledge Handbook of the Ethics of Discrimination, hrsg. von Kasper Lippert-Rasmussen, 30-41. London. 
Knight, Carl. 2017. „Discrimination and equality of opportunity“. In The Routledge Handbook of the Ethics of Discrimination, hrsg. von Kasper Lippert-Rasmussen, 140-150. London.

Lazenby, Hugh, und Paul Butterfield. 2017. „Discrimination and the personal sphere“. In The Routledge Handbook of the Ethics of Discrimination, hrsg. von Kasper Lippert-Rasmussen, 369-378. London.

Lippert-Rasmussen, Kasper. 2006. „The badness of discrimination“. Ethical Theory and Moral Practice 9, 167-185.

Lippert-Rasmussen, Kasper. 2007. „Nothing personal: on statistical discrimination“. The Journal of Political Philosophy 15(4), 385-403.

Lippert-Rasmussen, Kasper. 2009. „Discrimination and the aim of proportional representation“. Politics, Philosophy and Economics 7(2), 159-182.

Lippert-Rasmussen, Kasper. 2011. „We are all different': Statistical discrimination and the right to be treated as an individual“. Journal of Ethics 15(1), 47-59.

Lippert-Rasmussen, Kasper. 2012. „Intentions and Discrimination in Hiring“. Journal of Moral Philosophy 9, 55-74.

Lippert-Rasmussen, Kasper. 2014. Born Free and Equal? A Philosophical Inquiry into the Nature of Discrimination, Oxford.

Lippert-Rasmussen, Kasper, Hrsg. 2017. The Routledge Handbook of the Ethics of Discrimination. London.

Lippert-Rasmussen, Kasper. 2017a. „The philosophy of discrimination. An introduction“. In The Routledge Handbook of the Ethics of Discrimination, hrsg. von Kasper Lippert-Rasmussen, 1-16. London.

Lippert-Rasmussen, Kasper. 2017b. „Affirmative action, historical injustice, and the concept of beneficiaries“. Journal of Political Philosophy 25(1), 72-90.

Lübbe, Weyma. 2020. Corona-Triage. Ein Kommentar zu den anlässlich der Corona-Krise publizierten Triage-Empfehlungen der italienischen SIAARTI-Mediziner. https://verfassungsblog.de/corona-triage/, 21. April 2020.

Liu, Xiaofei. 2015. „No Fats, Femmes, or Asians“. Moral Philosophy and Politics 2(2), 255-276.

Mason, Andrew. 2017. „Appearance, discrimination, and reaction qualification“. Journal of Political Philosophy 25(1), 48-71.

Mengel, Friederike, Jan Sauermann und Ulf Zölitz. 2019. „Gender bias in teaching evaluations“. Journal of the European Economic Association 17(2), 535-566, DOI: $10.1093 /$ jeea/jvxo57.

Mikkola, Mari. 2017. „Discrimination and trans identities“. In The Routledge Handbook of the Ethics of Discrimination, hrsg. von Kasper Lippert-Rasmussen, 287297. London.

Moreau, Sophia. 2010. „What is discrimination?“. Philosophy and Public Affairs 38(2), 143-179. 
Rössler, Beate. 2012. „Frauen verzweifelt gesucht? Über Quoten und Gerechtigkeit“. Merkur 66(756), 371-381.

Scanlon, Thomas. 2008. Moral Dimensions. Permissibility, Meaning, Blame. Cambridge.

Schauer, Frederick. 2003. Profiles, Probabilities and Stereotypes. Cambridge.

Schauer, Frederick. 2017. „Statistical (and non-statistical) discrimination“. In The Routledge Handbook of the Ethics of Discrimination, hrsg. von Kasper Lippert-Rasmussen, 42-53. London.

Schouten, Gina. 2017. „Discrimination and gender“. In The Routledge Handbook of the Ethics of Discrimination, hrsg. von Kasper Lippert-Rasmussen, 185-195. London.

Segall, Shlomi. 2012. „What's so bad about discrimination?“. Utilitas 24(1), 83-100.

Selmi, Michael. 2013. „Indirect discrimination and the anti-discrimination mandate“. In Philosophical Foundations of Discrimination Law, hrsg. von Deborah Hellman und Sophia Moreau, 250-268. Oxford.

Sesardic, Neven, und Rafael De Clercq. 2014. „Women in philosophy: problems with the discrimination hypothesis“. Academic Questions 27, 461-473.

Slavny, Adam, und Tom Parr. 2016. „Harmless discrimination“. Legal Theory 21, 100-114.

Suk, Julie. 2017. „Discrimination and affirmative action“. In The Routledge Handbook of the Ethics of Discrimination, hrsg. von Kasper Lippert-Rasmussen, 394406. London.

Thomsen, Frej Klem. 2013. „But some groups are more equal than others: a critical review of the group-criterion in the concept of discrimination“. Social Theory and Practice 39(1), 120-146.

Thomsen, Frej Klem. 2017. Discrimination. Oxford Research Encyclopedia of Politics. http://politics.oxfordre.com/view/10.1093/acrefore/9780190228637.001.0001/ acrefore-9780190228637-e-202?print=pdf, 9. Juli 2017.

Uhlmann, Eric Luis, und Geoffrey L. Cohen. 2005. „Constructed criteria: redefining merit to justify discrimination“. Psychological Science 16(6), 474-480.

Valian, Virginia. 2005. „Beyond gender schemas: improving the advancement of women in academia“. Hypatia 20(3), 198-213.

Wasserman, David. 1998. „Discrimination, concept of“. In Encyclopedia of Applied Ethics (Band 1), hrsg. von R. Chadwick. 805-814. London.

Young, Iris M. 1990. Justice and the Politics of Difference, Princeton.

Young, Iris M. 2001. „Equality of whom? Social groups and judgments of justice“. The Journal of Political Philosophy 9(1), 1-18. 
